\title{
Collaboration can improve individual recognition memory: Evidence from immediate and delayed tests
}

\author{
Suparna Rajaram and Luciane P. Pereira-Pasarin \\ Stony Brook University, Stony Brook, New York
}

\begin{abstract}
In two experiments, we tested the effects of collaboration on individual recognition memory. In Experiment 1, participants studied pictures and words either for meaning or for surface properties and made recognition memory judgments individually either following group discussion among 3 members (collaborative condition) or in the absence of discussion (noncollaborative condition). Levels of processing and picture superiority effects were replicated, and collaboration significantly increased individual recognition memory. Experiment 2 replicated this positive effect and showed that even though memory sensitivity declined at longer delays ( $48 \mathrm{~h}$ and 1 week), collaboration continued to exert a positive influence. These findings show that (1) consensus is not necessary for producing benefits of collaboration on individual recognition, (2) collaborative facilitation on individual memory is robust, and (3) collaboration enhances individual memory further if conditions predispose individual accuracy in the absence of collaboration.
\end{abstract}

Individuals often retrieve the past in a group context. Although extensive research has accumulated on the cognitive processes that mediate individual memory, less is known about the influences of collaboration on the accuracy of individual memory. We report experiments designed to test this relationship.

The cognitive literature on collaborative memory is modest, and much of it has focused on group performance. Researchers have contrasted the product of a collaborative group with that of a nominal group consisting of individuals working alone, whose nonredundant responses were then pooled. Much of this evidence comes from free recall measures and reveals a collaborative inhibition effect, whereby recall of collaborative groups (of 3 members) is worse than that of nominal groups of equal size (Basden, Basden, Bryner, \& Thomas, 1997; Weldon \& Bellinger, 1997).

In contrast to its inhibitory effect on recall, collaboration has been shown to improve the accuracy of group performance in recognition memory (Clark, Hori, Putnam, \& Martin, 2000). This finding makes sense because recognition memory minimizes the role of a retrieval-blocking mechanism that is presumed to impair collaborative recall (Basden et al., 1997). However, a recognition memory task involves decisions on studied as well as nonstudied items, and the presence of nonstudied items can potentially increase false alarm responses in the group. Interestingly, Clark et al.'s findings showed a net benefit of collaboration in the form of increased memory sensitivity, or $d^{\prime}$.

Collaboration can influence memory in yet another important way - that is, by modulating the memory of the individual who engaged in group collaboration. Collaborative reconstruction of past events among individuals is an everyday experience; people discuss past company meetings, faculty meetings, or family vacations. Information from such collaborations must inevitably influence the quality, quantity, and accuracy of individual memory. In a handful of recent studies, the effects of prior collaboration have been examined; increased accuracy was reported in some cases, and decreased accuracy was reported in others. These studies typically used the sequential process of prior collaboration on later individual memory, whereas we focus on effects of collaboration on individual memory on a trial-by-trial basis, for reasons explained below. We first review the relevant studies to situate our work.

Positive influence of collaboration on later individual memory has been reported in free recall of words, pictures, and stories (Weldon \& Bellinger, 1997; see also Basden, Basden, \& Henry, 2000, and Basden, Reysen, \& Basden, 2002, for veridical memory conditions). In Weldon \& Bellinger's (1997, Experiment 1) study, participants studied two lists of unrelated words and performed two successive recalls (Recall 1 and Recall 2) in one of four combinations: (1) Recall 1 individually and Recall 2 individually (II), (2) Recall 1 collaboratively and Recall 2 collaboratively (CC), (3) Recall 1 individually and Recall 2 collaboratively (IC), and (4) Recall 1 collaboratively and Recall 2 individually (CI). Even though collaborative inhibition occurred at the group level, individual recall following collaboration (in the CI condition) was improved in comparison with the second of two individual recalls 
(in the II condition). These findings suggest that when study materials support veridical memory, prior collaboration improves individual memory.

In studies in which negative influences of collaboration on individual free recall (and also on individual recognition; Basden et al., 2002) were reported, the materials and conditions were conducive to increasing false memory. These studies included stimuli that typically increase individual false memory - for example, associatively related items or everyday scenes with typical items deleted (Basden et al., 2002; Roediger, Meade, \& Bergman, 2001). In these studies, false memories were also systematically introduced by the inclusion of either confederates who inserted incorrect responses during recall (Roediger et al., 2001) or input from a perceived group member that really consisted of experimenter-controlled, computer-generated responses (Basden et al., 2002). In fact, using a virtual confederate to manipulate collaboration can worsen later individual recognition memory even for word lists that are not susceptible to false memory effects (Reysen, 2005). Together, effects of collaboration on true and false memories suggest that collaboration magnifies individual memory output; when conditions encourage accurate memory in individuals, collaboration increases individual memory accuracy, but when conditions promote false memories in individuals, collaboration further increases individual memory errors.

As noted, in this article we focus on individual veridical memory. Much of our knowledge about the influence of collaboration comes from studies in which the free recall measure was used. Our interest was to probe this issue in a recognition memory task. In recall, participants provide retrieval cues, and it would seem that these cues can potentially trigger the memory of someone else in the group. But these cues appear to disrupt others' retrieval strategies and thereby decrease memory performance in collaborating groups (Basden et al., 1997; Wright \& Klumpp, 2004). In contrast, recognition memory performance does not depend on the cues provided by members of the group; therefore, an information-based exchange in this task is presumably the basis for collaborative facilitation in the group (Clark et al., 2000). In this situation, an incorrect "yes" response to a nonstudied item by one member can be corrected by another member, or omissions can be recovered similarly, before the group reports its consensus on each item.

However, if the requirement to reach a consensus is removed by asking participants to make individual recognition responses, these external corrections are not in play. In such situations, individual responses can be influenced by correct or incorrect input (more hits or more false alarms, respectively) received during collaborative discussion, or by both. As a result, individual memory sensitivity might improve, worsen, or remain the same, respectively. To test this issue, we conducted the recognition memory task, in which individual responses were made on each item either following group collaboration or in the absence of collaboration. The goal was to assess the effects of collaboration on individual recognition in the absence of a consensus requirement.
As was noted earlier, even though collaboration leads to reduction in group memory in free recall due to retrieval blocking, it nevertheless increases later individual recall (Weldon \& Bellinger, 1997). Therefore, in a task such as recognition, in which retrieval blocking is eliminated, the collaborative benefit should be present in individual performance. Thus, findings from free recall suggest that collaboration should increase accuracy in individual recognition. In contrast, the central findings from studies on false memory suggest that prior collaboration should increase either false alarms (Roediger, et al., 2001) or both hits and false alarms (Basden et al., 2002) in later individual recognition.

We favor the prediction of a net increase in memory sensitivity in our recognition study for the following reasons. First, we used standard stimuli (words and pictures) that do not usually create false memories. Second, retrieval blocking that reduces collaborative recall does not operate in recognition memory. Third, we did not manipulate collaboration through an experimenter-controlled, real or virtual confederate that systematically induces false alarms. Thus, our experiments consisted of optimal conditions for assessing whether or not information exchange during collaboration can improve individual recognition even in the absence of group consensus.

\section{EXPERIMENT 1}

\section{Method}

Participants. Sixty Stony Brook University undergraduates participated for research credit or payment of $\$ 10 / \mathrm{h}$.

Design and Materials. Following parts of Weldon and Bellinger's (1997, Experiment 1) methodology, we included two within-subjects variables at study: item type (pictures vs. words) and levels of processing (deep vs. shallow). A fifth condition (nonstudied) was included at test. Counterbalancing of item type resulted in 10 study lists. At test, collaboration/noncollaboration was manipulated between subjects, with 30 participants in each condition.

Ninety line drawings from Snodgrass and Vanderwart's (1980) norms were divided into five groups (pictures-deep, picturesshallow, words-deep, words-shallow, and nonstudied) of 18 items each. Grouped items were equated on word length (5-9 letters, $M=$ $6.23 ; F<1)$, frequency per million words $(M=14.48 ; F<1)$, and name agreement (i.e., the likelihood of naming each item correctly; $85 \%-100 \%, M=95.12 ; F<1)$. Recognition test booklets contained a cover page with instructions and 144 items presented in word form (72 critical studied, 18 critical nonstudied, and 54 fillers to achieve a studied:nonstudied ratio of 1:1). The letters Y ("yes"), N ("no"), R ("remember"), and K ("know") were listed next to each item. A sheet of paper was given to conceal all but one item at a time. A tape recorder was displayed to suggest recording of responses in the collaborative condition, to encourage participation by all group members.

Procedure. At study, all of the participants worked individually and received instructions auditorily from the experimenter and also in written form. Each trial included an asterisk $(2 \mathrm{sec})$ and a study item $(1 \mathrm{sec})$. Stimuli were projected on a white screen either as black line drawings or as lowercase words in Arial 40-point font.

The deep-versus-shallow manipulation was blocked at study, whereas pictures and words were randomly intermixed within each of these blocks. We adapted Weldon and Bellinger's (1997) levelsof-processing instructions and instructed the participants to rate the pleasantness of each item in the real world $(1=$ very unpleasant, $5=$ very pleasant) for deep encoding, and the sharpness of the word font or the graphic quality of pictures $(1=$ very unclear, $5=$ very clear $)$ 
for shallow encoding. The font or graphic quality did not vary across items, but, as in Weldon and Bellinger, these instructions were used to direct the participants to process information at a shallow level. During the 1:10-h delay, the participants played computer solitaire for $20 \mathrm{~min}$, worked individually on a 1,000-piece jigsaw puzzle for $40 \mathrm{~min}$, and, finally, received instructions for the recognition test, remember/know judgments, and collaborative discussion (if applicable).

The remember/know instructions were adapted from Rajaram (1993). The participants were instructed to circle the letter R if they had a vivid recollection of the item from the study phase and the letter $\mathrm{K}$ if they were confident they had seen the item during study but were unable to recall specific details about the item from study. Past studies on collaboration have sometimes included the remember/ know measure but reported mixed patterns. In some cases, collaboration increased remember/know judgments for both false memories (Basden, Basden, Thomas, \& Souphasith, 1998) and true memories (Basden et al., 2002), and in other cases collaboration increased only know judgments for false memories (Roediger, et al., 2001). We included the remember/know paradigm in this experiment to explore whether or not information received from other group members would differentially modulate remembering and knowing in our design. On one hand, individuals might retrieve specific details while attempting to convince other members of their decisions (see Clark et al., 2000, for a similar idea), or they might be reminded of specific details when other members contribute responses. This process would increase remember responses in the collaborative condition. On the other hand, although individuals might be persuaded by other members about an item's study status, they might not share others' vivid recollections or be able to invoke specific details for the item. This process would increase know responses instead. It is also possible that collaboration does not selectively increase one of these two subjective experiences but influences both, for the reasons just described. If this is the case, differential effects of collaboration on remembering or knowing would not be discernible.
At test, in the collaborative condition 3 participants worked together, and in the noncollaborative condition the participants worked individually. The participants in the collaborative condition were instructed to work together and discuss each item. After the group discussion of each item, the participants indicated individually whether or not they recognized that item from the study list and, when applicable, made remember/know judgments before the group proceeded to the next item. The participants were explicitly told that their individual responses were of main interest regardless of whether or not the responses concurred with the group discussion. The recognition test was self-paced. During collaboration, the participants produced their responses aloud. The discussion was brief and usually monosyllabic. Turn taking was not enforced, and free-flowing discussion was encouraged. The observations of the experimenter (L.P.P.-P.) revealed no discernible emergence of a "group leader."

The participants in the noncollaborative condition received the same test instructions except for the parts that pertained to collaboration. On the basis of pilot data, the participants in the noncollaborative condition were given $6 \mathrm{sec}$ to work on each item to equate the duration across the two groups. The study and test phases lasted approximately 7 and 15 min, respectively.

\section{Results and Discussion}

The alpha level was set at .05 in both experiments. Table 1 displays mean proportions of hits and false alarms, and Figure 1 displays the mean proportions of hits minus false alarms for overall recognition as a function of study and test conditions. For overall recognition, a $2 \times 2 \times 2$ mixed ANOVA on hits minus false alarms revealed a significant levels-of-processing effect (deep, $M=.71$; shallow, $M=.38)\left[F(1,58)=119.89, M S_{\mathrm{e}}=.05\right]$ and a significant picture superiority effect (pictures, $M=.58$;
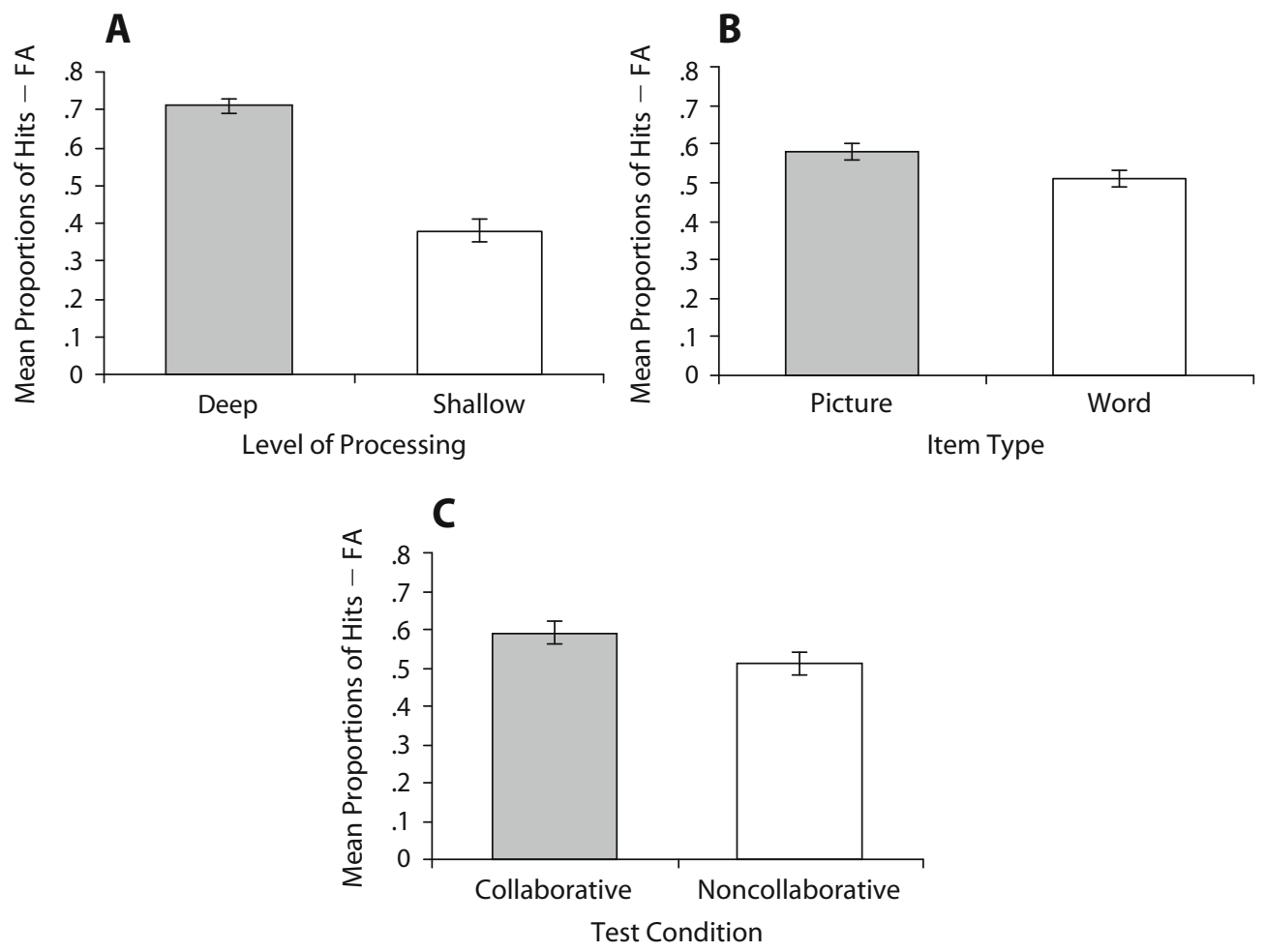

Figure 1. Mean proportions of hits minus false alarms for overall recognition as a function of levels of processing, item type, and collaborative versus noncollaborative conditions. 
Table 1

Mean Proportions of Hits and False Alarms for Overall Recognition

As a Function of Levels of Processing, Pictures Versus Words, and Collaborative Versus Noncollaborative Conditions

\begin{tabular}{|c|c|c|c|c|c|}
\hline \multirow[b]{3}{*}{ Condition } & \multicolumn{4}{|c|}{ Hits } & \multirow{3}{*}{$\begin{array}{c}\text { Nonstudied } \\
\text { (False Alarms }\end{array}$} \\
\hline & \multicolumn{2}{|c|}{ Pictures } & \multicolumn{2}{|c|}{ Words } & \\
\hline & Deep & Shallow & Deep & Shallow & \\
\hline Collaborative & .81 & .63 & .84 & .45 & .10 \\
\hline Noncollaborative & .87 & .58 & .87 & .43 & .18 \\
\hline
\end{tabular}

words, $M=.51)\left[F(1,58)=16.85, M S_{\mathrm{e}}=.02\right]$, replicating the standard effects in individual and collaborative memory (Weldon \& Bellinger, 1997). Neither item type $(F<1)$ nor levels of processing $\left[F(1,58)=1.75, M S_{\mathrm{e}}=\right.$ $.05]$ interacted with collaboration/noncollaboration.

Importantly, the effect of collaboration was significant: The participants in the collaborative condition $(M=$ $.59)$ outperformed those in the noncollaborative condition $(M=.51)\left[F(1,58)=4.29, M S_{\mathrm{e}}=.09\right]$. The benefit of collaboration was driven by a significant reduction in false alarms in the collaborative condition $[t(58)=2.11$, $S E=.04]$. The hit rates were equivalent (collaborative condition, $M=.68$; noncollaborative condition, $M=.69$; $F<1$ ). Importantly, hit rates were not reduced by collaboration, leading to a net benefit of collaboration without a trade-off between hits and false alarms.

For remember and know judgments, the false alarm rates were comparable in magnitude to those reported in the literature (for remember judgments, collaborative $=$ .07 , noncollaborative $=.08$; for know judgments, collaborative $=.03$, noncollaborative $=.10$ ). A $2 \times 2 \times 2$ mixed ANOVA on hits minus false alarms for remember judgments revealed a significant levels-of-processing effect (deep, $M=.53$; shallow, $M=.25)[F(1,58)=96.78$, $\left.M S_{\mathrm{e}}=.05\right]$ and a significant picture superiority effect (pictures, $M=.45$; words, $M=.33$ ) $[F(1,58)=26.21$, $\left.M S_{\mathrm{e}}=.04\right]$, replicating the typical findings in recognition studies (Gardiner, 1988; Rajaram, 1993). For know judgments, a small but significant levels-of-processing effect was observed (deep, $M=.18$; shallow, $M=.14$ ) $\left[F(1,58)=5.86, M S_{\mathrm{e}}=.02\right]$, for which we have no explanation. But, as we expected, there was a significant reversal of the picture superiority effect (pictures, $M=.13$; words, $M=.18)\left[F(1,58)=4.67, M S_{\mathrm{e}}=.03\right]$. The effect of collaboration was significant neither for remember judgments $(F<1)$ nor for know judgments $[F(1,58)=$ $\left.1.71, M S_{\mathrm{e}}=.10\right]$. Because, as in previous studies (Basden et al., 1998; Basden et al., 2002; Roediger et al., 2001), remember/know measures yielded inconclusive information, we do not discuss them further.

Returning to overall recognition, ${ }^{1}$ which was the main dependent measure of interest, the $d^{\prime}$ and $\beta$ analyses supported the patterns observed with the hits minus false alarms measure. Prior collaboration led to significantly higher $d^{\prime}$ than did noncollaboration (2.02 and 1.66, respectively) $[t(58)=2.21, S E=.16]$, and the $\beta$ values did not differ (collaborative, $\beta=4.62$; noncollaborative, $\beta=$ 3.43) $[t(58)=1.29, S E=.92]$.
In summary, collaboration improved individual recognition even when the consensus criterion was removed. Experiment 2 was conducted to replicate this positive effect and to assess its robustness as a function of test delay. The manipulation of test delay also provided an opportunity to evaluate a secondary question. Positive effects of collaboration on individual recognition in Experiment 1 (and on individual veridical recall in Weldon \& Bellinger, 1997) stand in contrast to the increase in individual false memory in other studies (Basden et al., 2002; Roediger et al., 2001). As was noted earlier, this contrasting pattern suggests that collaboration is a mechanism that boosts individual output - either in the direction of increased accuracy when experimental conditions induce veridical memories in individuals, or in the direction of decreased accuracy when conditions induce false memories. If this is the case, then collaboration should improve individual recognition memory even if individual veridical memory becomes weaker so long as hits exceed false alarms. Experiment 2 allowed a test of this additional issue because we used unrelated lists of items that do not activate strong schemas and therefore do not induce false memories. Test delay impairs recognition memory (Glanzer, Adams, \& Iverson, 1991; Tulving, Schacter, \& Stark, 1982) because hits decrease, false alarms increase, and old/new judgments become increasingly difficult with delay. If collaboration improves individual recognition only when veridical memories are strong, then the positive effect of collaboration should diminish with a decline in recognition memory across delay. However, if collaboration continues to exert a positive influence at longer delays, then the negative influence of collaboration likely results from the nature of the materials used at study and follows the direction of individual memory patterns.

\section{EXPERIMENT 2}

\section{Method}

The same method used in Experiment 1 was used in Experiment 2 except where noted.

Participants. Two hundred sixteen Stony Brook University students participated for course credit.

Design and Materials. A $2 \times 2 \times 3$ mixed-factorial design with one within-subjects variable (item type: pictures vs. words) and two between-subjects variables (test condition: collaborative vs. noncollaborative; delay: $1 \mathrm{~h}$ vs. $48 \mathrm{~h}$ vs. 1 week) was used, with 36 participants assigned to each of the six between-subjects conditions. Counterbalancing of item type (pictures vs. words vs. nonstudied) resulted in six study lists. 
The 90 critical items used in Experiment 1 were divided into three groups of 30 items each, equated for word length (5-9 letters, $M=$ $6.23 ; F<1)$, frequency per million words $(M=14.22 ; F<1)$, and name agreement $(85 \%-100 \%, M=95.04 ; F<1)$. Thirty additional fillers (at test) and 8 buffers ( 4 at study and 4 at test) were also equated to the 90 critical items on frequency $(M=14.45 ; F<1)$ and word length $(M=6.19 ; F<1)$. Pictures and words were blocked during study and presented on computer monitors. Blocks were counterbalanced for order, and each block contained 30 critical items, 1 primacy buffer, and 1 recency buffer. The recognition test booklet contained, randomly intermixed (except for buffers), 128 items in word form (60 critical studied, 30 critical nonstudied, 30 fillers, 4 studied buffers, and 4 nonstudied buffers) with the letters Y ("yes") and N ("no") presented next to each item.

Procedure. The same procedure used in Experiment 1 was used in the present experiment, with the following exceptions. The participants were asked to carefully view the pictures and read the words for an unspecified memory test. Recognition test instructions for all three delays were given just before the test phase. Remember/know judgments were not required.

\section{Results and Discussion}

As was expected, the false alarm rates increased with test delay ( $1 \mathrm{~h}, M=.18 ; 48 \mathrm{~h}, M=.34 ; 1$ week, $M=.45)$ $\left[F(2,210)=65.11, M S_{\mathrm{e}}=.02\right]$. As in Experiment 1, false alarms were significantly higher in the noncollaborative $(M=.36)$ than in the collaborative $(M=.29)$ condition $\left[F(1,210)=9.65, M S_{\mathrm{e}}=.02\right]$. Independent $t$ tests showed that this difference was significant at the $1-\mathrm{h}[t(70)=2.37$, $S E=.03]$ and 1-week $[t(70)=2.63, S E=.04]$ delay but, inexplicably, not at 48 -h delay $(t<1$; see Table 2$)$.

For the hits minus false alarms measure, a $2 \times 2 \times 3$ mixed ANOVA revealed a significant picture superiority effect (pictures, $M=.42$; words, $M=.25)[F(1,210)=$ $\left.278.83, M S_{\mathrm{e}}=.01\right]$ and a significant decline in recognition with delay $(1 \mathrm{~h}, M=.54 ; 48 \mathrm{~h}, M=.31 ; 1$ week, $M=$ .16) $\left[F(2,210)=138.74, M S_{\mathrm{e}}=.04\right]$. As was expected, in addition to the increase in false alarms, the decline in recognition performance across time was reflected in a $d e-$ crease in hits across delay $(1 \mathrm{~h}, M=.72 ; 48 \mathrm{~h}, M=.65$; 1 week, $M=.62)\left[F(2,210)=12.01, M S_{\mathrm{e}}=.04\right]$. The picture superiority effect was larger in the collaborative than in the noncollaborative condition $[F(1,210)=14.74$, $\left.M S_{\mathrm{e}}=.01\right]$ and declined over delay $[F(2,210)=8.53$,

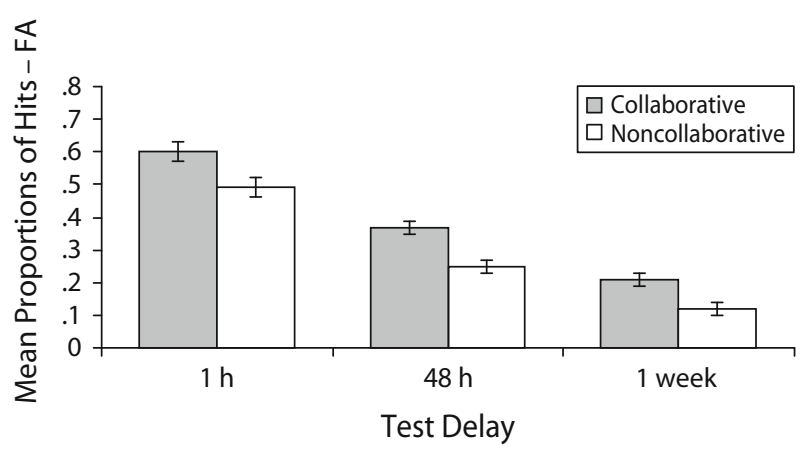

Figure 2. Mean proportions of hits minus false alarms as a function of collaborative versus noncollaborative conditions and retention interval.
Table 2

Mean Proportions of Hits and False Alarms, $d^{\prime}$, and $\beta$ As

a Function of Collaborative Versus Noncollaborative Test Condition and Test Delay

\begin{tabular}{|c|c|c|c|c|c|c|}
\hline \multirow[b]{3}{*}{ Response } & \multicolumn{6}{|c|}{ Test Delay } \\
\hline & \multicolumn{2}{|c|}{$1 \mathrm{~h}$} & \multicolumn{2}{|c|}{$48 \mathrm{~h}$} & \multicolumn{2}{|c|}{1 week } \\
\hline & $\mathrm{CO}$ & $\mathrm{NC}$ & $\mathrm{CO}$ & $\mathrm{NC}$ & $\mathrm{CO}$ & $\mathrm{NC}$ \\
\hline \multicolumn{7}{|l|}{ Hits } \\
\hline Pictures & .87 & .80 & .80 & .66 & .71 & .66 \\
\hline Words & .62 & .59 & .61 & .53 & .51 & .59 \\
\hline False alarms & .15 & .21 & .34 & .35 & .40 & .51 \\
\hline$d^{\prime}$ & 1.92 & 1.43 & 1.01 & 0.69 & 0.56 & 0.33 \\
\hline$\beta$ & 2.33 & 1.38 & 1.02 & 1.23 & 1.10 & 0.99 \\
\hline
\end{tabular}

Note- CO, collaborative condition; $\mathrm{NC}$, noncollaborative condition.

$\left.M S_{\mathrm{e}}=.01\right]$. However, the three-way interaction among item type, delay, and collaboration/noncollaboration was not significant $\left[F(2,210)=2.05, M S_{\mathrm{e}}=.01\right]$.

The critical finding from Experiment $1-\mathrm{a}$ benefit of collaboration on later individual memory - was replicated for both the hits minus false alarms measure and the $d^{\prime}$ measure. In the interest of economy, here we report only the $d^{\prime}$ analyses that showed significant advantages of collaboration (collaborative, $M=1.16$; noncollaborative, $M=.82)\left[F(1,210)=31.75, M S_{\mathrm{e}}=.20\right]$, significant disadvantages of delay $\left[F(2,210)=140.43, M S_{\mathrm{e}}=\right.$ $.20]$, and no decline in the collaboration advantage across delay $\left[F(2,210)=1.56, M S_{\mathrm{e}}=.20\right]$. Together, these findings show that positive effects of collaboration on later individual memory are replicable and remain preserved across delay.

The $\beta$ analyses showed that the participants used a more conservative criterion in the collaborative $(M=1.48)$ than in the noncollaborative $(M=1.20)$ condition $[F(1,210)=$ $\left.3.98, M S_{\mathrm{e}}=1.09\right]$ and that $\beta$ values declined significantly across delay $\left[F(2,210)=13.37, M S_{\mathrm{e}}=1.09\right]$. Nevertheless, the participants did not use a liberal response criterion at any of the delays. A significant interaction between collaboration and delay $\left[F(2,210)=5.95, M S_{\mathrm{e}}=1.09\right]$ was observed because $\beta$ values differed significantly between collaborative and noncollaborative conditions at 1-h delay $[t(70)=2.40, S E=.40]$ but not at $48-\mathrm{h}[t(70)=1.50$, $S E=.14]$ or 1 -week $[t(70)=1.65, S E=.07]$ delay.

Thus, positive influences of collaboration were found to be preserved even when memory sensitivity declined with delay. Across the two experiments, collaboration exerted a positive influence by reducing individual false alarm rates in three out of four conditions. Moreover, the net benefit from collaboration did not result from a trade-off between hits and false alarms at any delay.

\section{GENERAL DISCUSSION}

Two experiments were conducted to assess the effects of group collaboration on individual recognition memory through the use of study and test conditions that do not induce false memories. Experiment 1 revealed an increase in individual memory sensitivity following collabora- 
tion in comparison with no collaboration. This positive effect was replicated in Experiment 2 and was found to be significant after test delays of $48 \mathrm{~h}$ and even 1 week. These findings were observed in the context of simultaneous replication of the well-known effects of levels of processing, picture superiority, and decline in recognition memory with test delay.

These findings show an unequivocal advantage of collaboration on individual veridical memory. The positive effects reported here address the role of two factors in mediating effects of collaboration: the consensus criterion and memory sensitivity. Previous studies have shown that collaborative groups perform worse than nominal groups in recall (Basden et al., 1997; Weldon \& Bellinger, 1997). However, minimizing the effects of retrieval disruption through the use of a recognition task eliminates this collaborative inhibition and leads to collaborative facilitation in group recognition memory (Clark et al., 2000). The present experiments show that this collaborative advantage in recognition memory is preserved even when the consensus criterion needed for producing a single group response is removed.

At the individual level, collaboration has been shown to increase individual false recall and recognition when study materials induced false memories (Basden et al., 2002; Roediger et al., 2001). By implication, other experimental conditions that also decrease the accuracy of individual memory should make individuals vulnerable to accepting erroneous input from other group members. However, the present findings show that reduced memory sensitivity is not necessarily diagnostic of the direction in which collaboration influences individual memory. Even when overall recognition memory declined at 1-week test delay and false alarms increased, collaboration continued to improve individual recognition memory. Our findings show that the effects of collaboration on individual memory amplify the positive or negative effects that emerge in the absence of collaboration.

\section{AUTHOR NOTE}

We thank Mary Sue Weldon for helpful discussions during the early stages of this project. Parts of the research reported in this article were presented at the 77th Annual Meeting of the Eastern Psychological Association, Baltimore. Correspondence concerning this article should be addressed to S. Rajaram, Department of Psychology, Stony Brook University, Stony Brook, NY 11794-2500 (e-mail: suparna.rajaram@ sunysb.edu).

\section{REFERENCES}

Basden, B. H., Basden, D. R., Bryner, S., \& Thomas, R. L., III (1997). A comparison of group and individual remembering: Does collaboration disrupt retrieval strategies? Journal of Experimental Psychology: Learning, Memory, \& Cognition, 23, 1176-1189.

Basden, B. H., Basden, D. R., \& Henry, S. (2000). Costs and benefits of collaborative remembering. Applied Cognitive Psychology, 14, 497-507.

Basden, B. H., Basden, D. R., Thomas, R. L., III, \& Souphasith, S. (1998). Memory distortion in group recall. Current Psychology: Developmental, Learning, Personality, Social, 16, 225-246.

Basden, B. H., Reysen, M. B., \& Basden, D. R. (2002). Transmitting false memories in social groups. American Journal of Psychology, 115, 211-231.

Clark, S. E., Hori, A., Putnam, A., \& Martin, T. P. (2000). Group collaboration in recognition memory. Journal of Experimental Psychology: Learning, Memory, \& Cognition, 26, 1578-1588.

Gardiner, J. M. (1988). Functional aspects of recollective experience. Memory \& Cognition, 16, 309-313.

Glanzer, M., Adams, J. K., \& Iverson, G. (1991). Forgetting and the mirror effect in recognition memory: Concentering of underlying distributions. Journal of Experimental Psychology: Learning, Memory, \& Cognition, 17, 81-93.

RAJARAM, S. (1993). Remembering and knowing: Two means of access to the personal past. Memory \& Cognition, 21, 89-102.

Reysen, M. B. (2005). The effects of conformity on recognition judgments. Memory, 13, 87-94.

Roediger, H. L., III, Meade, M. L., \& Bergman, E. T. (2001). Social contagion of memory. Psychonomic Bulletin \& Review, 8, 365-371.

SnOdGrass, J. G., \& VANDERWART, M. (1980). A standardized set of 260 pictures: Norms for name agreement, image agreement, familiarity, and visual complexity. Journal of Experimental Psychology: Human Learning \& Memory, 6, 174-215.

Tulving, E., Schacter, D. L., \& Stark, H. A. (1982). Priming effects in word-fragment completion are independent of recognition memory. Journal of Experimental Psychology: Human Learning \& Memory, 8, 336-342.

Weldon, M. S., \& Bellinger, K. D. (1997). Collective memory: Collaborative and individual processes in remembering. Journal of Experimental Psychology: Learning, Memory, \& Cognition, 23, 1160-1175.

Wright, D. B., \& Klumpr, A. (2004). Collaborative inhibition is due to the product, not the process, of recalling in groups. Psychonomic Bulletin \& Review, 11, 1080-1083.

\section{NOTE}

1. The $d^{\prime}$ and $\beta$ values in both experiments were calculated on the overall recognition scores collapsed across item type (Experiments 1 and 2) and levels of processing (Experiment 1) in order to focus on the comparison of main theoretical interest: the effect of collaboration on individual recognition memory.

(Manuscript received December 23, 2005; revision accepted for publication May 25, 2006.) 